\title{
A posteriori estimates for errors of functionals on finite volume approximations to solutions of elliptic boundary value problems
}

\author{
Lutz Angermann*
}

\begin{abstract}
This article describes the extension of recent methods for a posteriori error estimation such as dual-weighted residual methods to node-centered finite volume discretizations of second order elliptic boundary value problems including upwind discretizations. It is shown how different sources of errors, in particular modeling errors and discretization errors, can be estimated with respect to a user-defined output functional.

Keywords: Finite volume methods, a posteriori error estimates, DWR method, goal-oriented estimation

2010 Mathematics Subject Classification: 65 N 08, 65 N 15, 65 N 30, 65 N 50
\end{abstract}

\section{Introduction}

In many areas of practical interest, e.g. computational fluid dynamics or image reconstruction, the computations are complicated and expensive, effectively limiting the achievable precision. In order to overcome these problems, adaptive finite element approaches are in use since several decades (see, e.g., BR78]). For instance, in the so-called $h$-adaptive methods the computational meshes are refined locally so that the mesh captures the variation of the solution while remaining coarse elsewhere. It has been shown that such approaches are computationally much more efficient than uniform meshes. In recent years, there has been considerable progress in applying these techniques to more involved questions such as the a posteriori error estimation of values of nonlinear functionals of interest (goal-oriented estimation, see, e.g., [BR01, [BR03, [Ran05]) or the (additional) a posteriori estimation of modeling errors (see, e.g., OV00], [VO01, [BE03]).

The present paper describes the extension of recent techniques for obtaining a posteriori error estimates for modeling and discretization errors to nonlinear second-order elliptic PDEs which are discretized by means of node-centered finite volume schemes including stabilization mechanisms of upwind type. Finite volume methods are attractive methods in selected areas of application, and therefore it is a natural requirement to develop analogous methods of error control for FVM. However, since finite volume methods suffer, in general, from the so-called property of Galerkin-orthogonality, special attention is to be paid to the treatment of the resulting defect term. It is shown that the extension of the dual-weighted a posteriori error estimates to finite volume discretizations is possible in a reasonable way. Furthermore, the latter approach is interesting because of the fact that different sources of errors (i.e. not only discretization errors but, for example, also modeling errors) can be estimated with respect to a

${ }^{*}$ Technische Universität Clausthal, Institut für Mathematik, Erzstr. 1, D-38678 Clausthal-Zellerfeld, Federal Republic of Germany, e-mail: lutz.angermann@tu-clausthal.de 
rather arbitrary user-defined output functional. For instance, in the field of inverse problems, the Tikhonov functionals can serve as typical output functionals (see, e.g., [BK10]).

Here we will mainly deal with Voronoi and Donald finite volume partitions on simplicial primary partitions of the computational domain; however the ideas can be extended to more general primary partitions, in particular quadrilateral or hexahedral partitions (cf., e.g., Ang06, Sect. 4.2]).

We consider the following boundary value problem with respect to the unknown function $u: \Omega \rightarrow \mathbb{R}:$

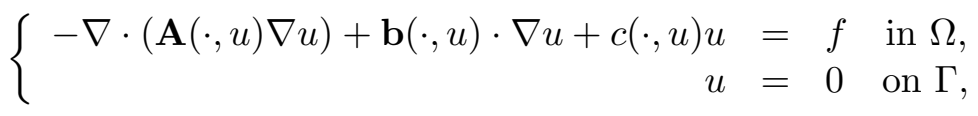

where $\Omega \subset \mathbb{R}^{d}, d \in\{2,3\}$, is a bounded polygonal or polyhedral domain with a Lipschitzian boundary $\Gamma$, and the data in (11) are sufficiently smooth:

$$
\mathbf{A}: \Omega \times \mathbb{R} \rightarrow \mathbb{R}^{d, d}, \quad \mathbf{b}: \Omega \times \mathbb{R} \rightarrow \mathbb{R}^{d}, \quad c: \Omega \times \mathbb{R} \rightarrow \mathbb{R}, \quad f: \Omega \rightarrow \mathbb{R} .
$$

Equations of such type may occur in various areas of science, for example in the mathematical description of filtration processes in nonhomogeneous media.

Using the formal notation

$$
\begin{aligned}
(w, v) & :=\int_{\Omega} w v d x \\
(\nabla w, \nabla v) & :=\int_{\Omega} \nabla w \cdot \nabla v d x \\
b(w ; v) & :=\frac{1}{2}[(\mathbf{b}(\cdot, w) \cdot \nabla w, v)-(w, \mathbf{b}(\cdot, w) \cdot \nabla v)], \\
d(w ; v) & :=(c(\cdot, w) w, v)-\frac{1}{2}(w \nabla \cdot \mathbf{b}(\cdot, w), v), \\
a(w ; v) & :=(\mathbf{A}(\cdot, w) \nabla w, \nabla v)+b(w ; v)+d(w ; v),
\end{aligned}
$$

and $\langle f, v\rangle:=(f, v)$, the variational formulation of the problem (11) in the space $V:=H_{0}^{1}(\Omega)$ reads as follows:

Find $u \in V$ such that

$$
\forall v \in V: \quad a(u ; v)=\langle f, v\rangle .
$$

Regarding results for the existence, uniqueness and regularity of solutions of (11) or (4), there is a wide literature both of relatively general nature (see, e.g., [B0̈8, Ch. 2] for a short survey) as well as for more specialized equations (see, e.g., [AS06]).

\section{The finite volume scheme}

Finite volume methods are attractive discretization methods for partial differential equations of first or second order in conservative form since they adequately transfer the conservation law, which is expressed by the differential equation, to the discrete level. At the same time, due to their proximity to finite difference methods, they are relatively easy to implement even in the nonlinear situation. However, a drawback of many finite volume methods is that there is no $p$-hierarchy as in finite element methods, therefore the order of accuracy (related to the 


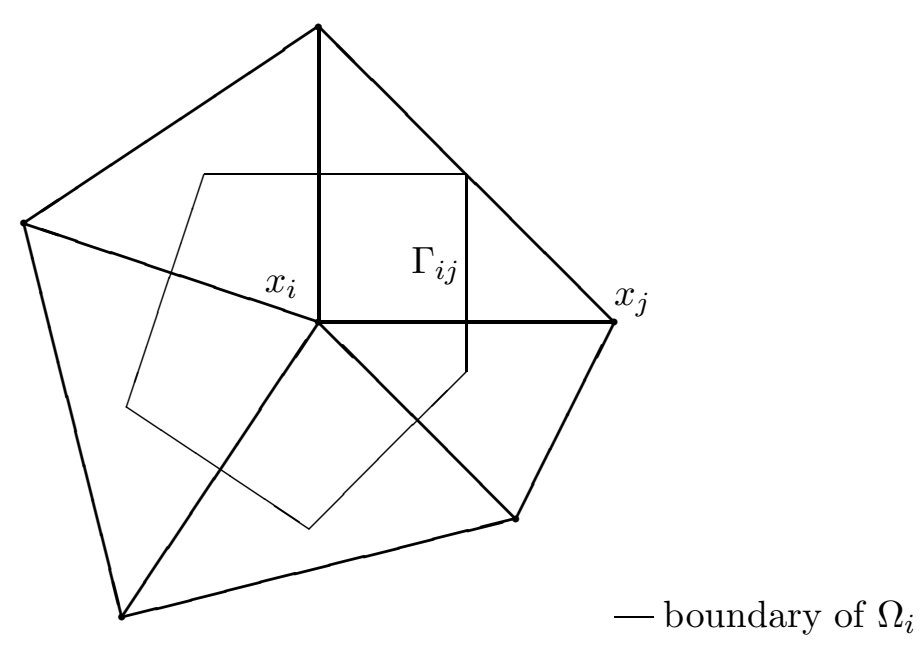

Figure 1: Configuration for the Voronoi-type discretization $(d=2)$

grid size) is relatively low. Nevertheless finite volume methods find wide applications in the computational practice. A certain degree of compensation can be achieved by the application of adaptive techniques based on a posteriori error estimates, as discussed in the subsequent section.

In this section we concentrate on node-centered finite volume methods for the discretization of problem (11).

\subsection{The case of Voronoi diagrams and scalar diffusion coefficients}

Let us consider a family of Voronoi diagrams such that their straight-line duals are Delaunay triangulations of $\Omega$ consisting of self-centered simplices. Here a simplex $T$ is called self-centered if its circumcentre lies in the interior of $T$ or on the boundary $\partial T$.

Denote by $\bar{\Lambda} \subset \mathbb{N}$ the index set of all vertices $x_{i}$ of a particular triangulation $\mathcal{T}$ and by $\Lambda \subset \bar{\Lambda}$ the index set of all vertices lying in $\Omega$.

In more detail, let

$$
\begin{aligned}
& \Omega_{i}:=\Omega_{i}^{V}:=\left\{x \in \Omega:\left\|x-x_{i}\right\|<\left\|x-x_{i}\right\| \forall j \in \bar{\Lambda} \backslash\{i\}\right\}, i \in \bar{\Lambda}, \\
& \text { where }\|\cdot\| \text { denotes the Euclidean norm in } \mathbb{R}^{d} \text {, } \\
& m_{i}:=\operatorname{meas}_{d}\left(\Omega_{i}\right) \text {, } \\
& \text { where } \operatorname{meas}_{d}(\cdot) \text { denotes the } d \text {-dimensional volume, } \\
& \Gamma_{i j}:=\partial \Omega_{i} \cap \partial \Omega_{j}, \Gamma_{i j}^{T}:=\Gamma_{i j} \cap T, i \in \Lambda, j \in \bar{\Lambda} \backslash\{i\}, T \in \mathcal{T}, \\
& m_{i j}:=\operatorname{meas}_{d-1}\left(\Gamma_{i j}\right), m_{i j}^{T}:=\operatorname{meas}_{d-1}\left(\Gamma_{i j}^{T}\right) \text {, } \\
& d_{i j}:=\left\|x_{i}-x_{j}\right\| \text {, } \\
& \Lambda_{i}:=\left\{j \in \bar{\Lambda} \backslash\{i\}: m_{i j} \neq 0\right\}, \\
& \Lambda_{T}:=\left\{i \in \bar{\Lambda}: x_{i} \in \partial T\right\} \text {, } \\
& h:=\max _{T \in \mathcal{T}} h_{T}, \quad \text { where } h_{T}:=\operatorname{diam} T \text {. }
\end{aligned}
$$


The finite volume solution will be interpolated in the discrete space

$$
V_{\mathcal{T}}:=\left\{v \in V:\left(\forall T \in \mathcal{T}:\left.v\right|_{T} \in \mathcal{P}_{1}(T)\right)\right\}
$$

where $\mathcal{P}_{1}(T)$ is the set of all first degree polynomials on $T$. We introduce a so called lumping operator

$$
L_{\mathcal{T}}: C(\bar{\Omega}) \rightarrow L_{\infty}(\Omega) \quad \text { acting as } \quad L_{\mathcal{T}} v:=\sum_{i \in \bar{\Lambda}} v\left(x_{i}\right) \chi_{\Omega_{i}}
$$

where $\chi_{\Omega_{i}}$ denotes the indicator function of the set $\Omega_{i}$.

Due to stability reasons, especially for the case of dominating convection, the class of finite volume methods under consideration is characterized by an additional stabilization technique called upwinding. For that purpose we introduce a scaling function $K: \mathbb{R} \rightarrow[0, \infty)$ which is defined by the help of a weighting function $r: \mathbb{R} \rightarrow[0,1]$ as $K(z):=1-[1-r(z)] z$.

A typical example of such a weighting function is

$$
r(z):=1-\frac{1}{z}\left(1-\frac{z}{e^{z}-1}\right)
$$

leading to $K(z)=z /\left(e^{z}-1\right)$, the Bernoulli function.

The discrete problem for the case of a scalar diffusion coefficient, i.e. where $\mathbf{A}$ is of the form $A \mathbf{I}$ with $A: \Omega \times \mathbb{R} \rightarrow \mathbb{R}$ and $\mathbf{I}$ being the identity in $\mathbb{R}^{d}$, is formulated as follows:

Find $u_{\mathcal{T}} \in V_{\mathcal{T}}$ such that

$$
\forall v_{\mathcal{T}} \in V_{\mathcal{T}}: \quad a_{\mathcal{T}}\left(u_{\mathcal{T}} ; v_{\mathcal{T}}\right)=\left\langle f_{\mathcal{T}}, v_{\mathcal{T}}\right\rangle
$$

where

$$
\begin{aligned}
a_{\mathcal{T}}\left(w_{\mathcal{T}} ; v_{\mathcal{T}}\right) & :=\sum_{i \in \Lambda} v_{\mathcal{T} i}\left\{\sum_{j \in \Lambda_{i}} \frac{\mu_{i j}}{d_{i j}} K\left(\frac{\gamma_{i j} d_{i j}}{\mu_{i j}}\right)\left(w_{\mathcal{T} i}-w_{\mathcal{T} j}\right) m_{i j}+c_{i} w_{\mathcal{T} i} m_{i}\right\} \\
\left\langle f_{\mathcal{T}}, v_{\mathcal{T}}\right\rangle & :=\sum_{i \in \Lambda} f_{i} v_{\mathcal{T} i} m_{i}
\end{aligned}
$$

and

$$
\begin{aligned}
\mu_{i j}=\mu_{i j}\left(w_{\mathcal{T} i}, w_{\mathcal{T} j}\right) & :=A\left(\frac{x_{i}+x_{j}}{2}, \frac{w_{\mathcal{T} i}+w_{\mathcal{T} j}}{2}\right) \\
\gamma_{i j}=\gamma_{i j}\left(w_{\mathcal{T} i}, w_{\mathcal{T} j}\right) & :=\nu_{i j} \cdot \mathbf{b}\left(\frac{x_{i}+x_{j}}{2}, \frac{w_{\mathcal{T} i}+w_{\mathcal{T} j}}{2}\right) \\
c_{i}=c_{i}\left(w_{\mathcal{T} i}\right) & :=c\left(x_{i}, w_{\mathcal{T} i}\right), \quad f_{i}:=f\left(x_{i}\right) .
\end{aligned}
$$

Moreover, we introduce the following norms and seminorms on $V_{\mathcal{T}}$ :

$$
\begin{aligned}
\left\|v_{\mathcal{T}}\right\|_{\mathcal{T}} & :=\sqrt{\left(v_{\mathcal{T}}, v_{\mathcal{T}}\right)_{\mathcal{T}}}=\left\|L_{\mathcal{T}} v_{\mathcal{T}}\right\|_{0,2, \Omega} \\
\left|v_{\mathcal{T}}\right|_{V} & :=\left\{\sum_{i \in \Lambda} v_{\mathcal{T} i} \sum_{j \in \Lambda_{i}}\left(v_{\mathcal{T} i}-v_{\mathcal{T} j}\right) \frac{m_{i j}}{d_{i j}}\right\}^{1 / 2} \\
\left\|v_{\mathcal{T}}\right\|_{V} & :=\left\{\left|v_{\mathcal{T}}\right|_{V}^{2}+\left\|v_{\mathcal{T}}\right\|_{\mathcal{T}}^{2}\right\}^{1 / 2}
\end{aligned}
$$


For the sake of consistency in the notations, we also use the following abbreviations of wellknown seminorms and norms in the Sobolev space $H^{1}(\Omega)$ :

$$
\left|v_{\mathcal{T}}\right|_{D}:=\left|v_{\mathcal{T}}\right|_{1,2, \Omega}, \quad\left\|v_{\mathcal{T}}\right\|_{D}:=\left\|v_{\mathcal{T}}\right\|_{1,2, \Omega} .
$$

The scheme (6) with the weighting function (5) is often called exponentially upwinded. It can be defined for other control functions $r: \mathbb{R} \rightarrow[0,1]$, too. However, we have to assume that all of these control functions satisfy the following properties:

(P1) $\quad r(z)$ is monotone for all $z \in \mathbb{R}$,

(P2) $\quad \lim _{z \rightarrow-\infty} r(z)=0, \quad \lim _{z \rightarrow \infty} r(z)=1$,

(P3) $1+z r(z) \geq 0 \quad$ for all $z \in \mathbb{R}$,

(P4) $\quad[1-r(z)-r(-z)] z=0 \quad$ for all $z \in \mathbb{R}$,

$$
\left[r(z)-\frac{1}{2}\right] z \geq 0 \quad \text { for all } z \in \mathbb{R}
$$

(P6) $\quad z r(z)$ is Lipschitz-continuous for all $z \in \mathbb{R}$.

We get from $(\underline{\mathrm{P} 4})$ the relation

$$
1+z r(z)=K(-z) .
$$

Replacing in (77) the argument $z$ by $-z$, (표 $)$ immediately implies

$$
K(z) \geq 0 \quad \text { for all } z \in \mathbb{R} .
$$

EXAMPLE 1 The function

$$
r(z)=\frac{1}{2}[\operatorname{sign} z+1],
$$

due to [BT81], has been investigated in [Ris86], [Ris90] for a linear equation in [1). This scheme is called fully upwinded.

The next two examples are simple approximations of (5).

EXAMPLE 2

$$
r(z)=\left\{\begin{array}{ccccc}
0 & , & z & < & -m \\
\frac{z+m}{2 m} & , & |z| & \leq & m
\end{array}, \quad 0<m \leq 8,\right.
$$

\section{EXAMPLE 3}

$$
r(z)=\left\{\begin{array}{ccccc}
0 & , & z & < & -m \\
0.5 & , & |z| & \leq & m \\
1 & , & z & > &
\end{array} \quad 0 \leq m \leq 2 .\right.
$$

This function violates property (P6).

EXAMPLE 4 The choice of the function

$$
r(z)=\frac{1}{2}\left[\frac{z}{2+|z|}+1\right]
$$

goes back to Samarskij [Sam65]. 
EXAMPLE 5 In $\left[M C L^{+} 83\right]$ the function

$$
r(z)=\frac{1}{2}[\tanh z+1]
$$

was proposed.

EXAMPLE 6 Finally, it can be taken the function

$$
r(z)=\left\{\begin{array}{ll}
(1-\sigma) / 2 & , \quad z<0 \\
(1+\sigma) / 2 & , \quad z \geq 0
\end{array} \quad \text { with } \quad \sigma(z):=\max \left\{0,1-\frac{2}{|z|}\right\}\right.
$$

what corresponds to Ikeda's partial upwind scheme E [Ike83].

Both for computational and theoretical reasons it may be advisable, in a really nonlinear situation, to choose differentiable control functions $r$. In the sequel, if there is no special reference, we assume that the scheme under consideration is defined for a general function $r$ that possesses the properties ( $(\overline{\mathrm{P} 1})$ to $(\overline{\mathrm{P} 6})$.

Finally we mention two equivalent representations of the form $a_{\mathcal{T}}$. First we remember that the leading coefficient $\frac{\mu_{i j}}{d_{i j}} K\left(\frac{\gamma_{i j} d_{i j}}{\mu_{i j}}\right)$ in $a_{\mathcal{T}}$ can be written, by the definition of $K$, in the following manner:

$$
\frac{\mu_{i j}}{d_{i j}} K\left(\frac{\gamma_{i j} d_{i j}}{\mu_{i j}}\right)=\frac{\mu_{i j}}{d_{i j}}\left\{1-\frac{\gamma_{i j} d_{i j}}{\mu_{i j}}\left[1-r\left(\frac{\gamma_{i j} d_{i j}}{\mu_{i j}}\right)\right]\right\}=\frac{\mu_{i j}}{d_{i j}}-\left(1-r_{i j}\right) \gamma_{i j},
$$

where $r_{i j}:=r\left(\frac{\gamma_{i j} d_{i j}}{\mu_{i j}}\right)$. Hence we get the representation

$$
\begin{gathered}
\sum_{i \in \Lambda} v_{\mathcal{T} i}\left\{\sum_{j \in \Lambda_{i}}\left\{v_{\mathcal{T}}\right)\right. \\
\left.+c_{i j}\left(w_{\mathcal{T} i}-w_{\mathcal{T} i} m_{i}\right) \frac{m_{i j}}{d_{i j}}-\left(1-r_{i j}\right)\left(w_{\mathcal{T} i}-w_{\mathcal{T} j}\right) \gamma_{i j} m_{i j}\right\} \\
\end{gathered}
$$

Furthermore, introducing the notations

$$
\begin{aligned}
a_{\mathcal{T}}^{0}\left(w_{\mathcal{T}} ; v_{\mathcal{T}}\right) & :=\sum_{i \in \Lambda} v_{\mathcal{T} i} \sum_{j \in \Lambda_{i}} \mu_{i j}\left(w_{\mathcal{T} i}-w_{\mathcal{T} j}\right) \frac{m_{i j}}{d_{i j}} \\
b_{\mathcal{T}}\left(w_{\mathcal{T}} ; v_{\mathcal{T}}\right) & :=\sum_{i \in \Lambda} v_{\mathcal{T} i} \sum_{j \in \Lambda_{i}}\left[\left(1-r_{i j}\right) w_{\mathcal{T} j}-\left(\frac{1}{2}-r_{i j}\right) w_{\mathcal{T} i}\right] \gamma_{i j} m_{i j} \\
d_{\mathcal{T}}\left(w_{\mathcal{T}} ; v_{\mathcal{T}}\right) & :=\sum_{i \in \Lambda}\left\{c_{i} m_{i}-\frac{1}{2} \sum_{j \in \Lambda_{i}} \gamma_{i j} m_{i j}\right\} w_{\mathcal{T}_{i}} v_{\mathcal{T}_{i}}
\end{aligned}
$$

we get a splitting of $a_{\mathcal{T}}$ which is comparable with (3):

$$
a_{\mathcal{T}}\left(w_{\mathcal{T}} ; v_{\mathcal{T}}\right)=a_{\mathcal{T}}^{0}\left(w_{\mathcal{T}} ; v_{\mathcal{T}}\right)+b_{\mathcal{T}}\left(w_{\mathcal{T}} ; v_{\mathcal{T}}\right)+d_{\mathcal{T}}\left(w_{\mathcal{T}} ; v_{\mathcal{T}}\right)
$$


REMARK 1 In the special case $\nabla \cdot \mathbf{b} \equiv 0$ on $\Omega$, it is senseful to use the following versions of $b_{\mathcal{T}}$ and $d_{\mathcal{T}}$ :

$$
\begin{aligned}
b_{\mathcal{T}}\left(w_{\mathcal{T}} ; v_{\mathcal{T}}\right) & =\sum_{i \in \Lambda} v_{\mathcal{T} i} \sum_{j \in \Lambda_{i}}\left[\left(1-r_{i j}\right) w_{\mathcal{T} j}+r_{i j} w_{\mathcal{T} i}\right] \gamma_{i j} m_{i j} \\
d_{\mathcal{T}}\left(w_{\mathcal{T}} ; v_{\mathcal{T}}\right) & =\sum_{i \in \Lambda} c_{i} w_{\mathcal{T} i} v_{\mathcal{T}_{i}} m_{i}
\end{aligned}
$$

\subsection{The case of Voronoi diagrams and matrix-valued diffusion coefficients}

Using the representation

$$
w_{\mathcal{T}}=\sum_{j \in \Lambda_{T}} w_{\mathcal{T} j} \psi_{j}
$$

on a single element $T$, where $\left\{\psi_{j}\right\}_{j \in \Lambda}$ is the standard nodal basis of $V_{\mathcal{T}}$, we easily see that

$$
\begin{aligned}
\int_{\partial \Omega_{i} \cap T}\left(\mathbf{A} \nabla w_{\mathcal{T}}\right) \cdot \nu d s & =\sum_{j \in \Lambda_{T}} \int_{\partial \Omega_{i} \cap T} w_{\mathcal{T} j}\left(\mathbf{A} \nabla \psi_{j}\right) \cdot \nu d s \\
& =\sum_{j \in \Lambda_{T} \backslash\{i\}}\left(w_{\mathcal{T} j}-w_{\mathcal{T} i}\right) \int_{\partial \Omega_{i} \cap T}\left(\mathbf{A} \nabla \psi_{j}\right) \cdot \nu d s
\end{aligned}
$$

In the next step the matrix $\mathbf{A}$ is approximated by a piecewise constant matrix $\mathbf{A}_{\mathcal{T}}$. Summing up over all elements $T$ which lie in the support of $\psi_{i}$, we have the relation

$$
\int_{\Omega}\left(\mathbf{A}_{\mathcal{T}} \nabla w_{\mathcal{T}}\right) \cdot \nabla \psi_{i} d x=\sum_{j \in \Lambda_{i}}\left(w_{\mathcal{T} i}-w_{\mathcal{T} j}\right) \int_{\partial \Omega_{i}}\left(\mathbf{A}_{\mathcal{T}} \nabla \psi_{j}\right) \cdot \nu d s
$$

With the definition

$$
\mu_{i j}:= \begin{cases}\frac{d_{i j}}{m_{i j}} \int_{\partial \Omega_{i}}\left(\mathbf{A} \nabla \psi_{j}\right) \cdot \nu d s, & m_{i j}>0 \\ 0, & m_{i j}=0\end{cases}
$$

it follows that

$$
\int_{\Omega}\left(\mathbf{A} \nabla w_{\mathcal{T}}\right) \cdot \nabla \psi_{i} d x \approx \int_{\Omega}\left(\mathbf{A}_{\mathcal{T}} \nabla w_{\mathcal{T}}\right) \cdot \nabla \psi_{i} d x=\sum_{j \in \Lambda_{i}} \mu_{i j}\left(w_{\mathcal{T} i}-w_{\mathcal{T} j}\right) \frac{m_{i j}}{d_{i j}} .
$$

Unfortunately, it is wellknown that in the case $d=3$ the right equality does not hold. Nevertheless, the right-hand side - together with the above definition (12) of $\mu_{i j}$ - is senseful for $d=3$, and thus this formula can be used for discretization.

Consequently, in order to obtain a discretization for the case of a matrix-valued diffusion coefficient, it is sufficient to replace in the forms $a_{\mathcal{T}}^{0}$ and $b_{\mathcal{T}}$ the corresponding values of $\mu_{i j}$ according to formula (12).

REMARK 2 The really critical point in the discretization of diffusion-convection equations with matrix-valued diffusion coefficients consists in the appropriate choice of the stabilization mechanism in the situation where the eigenvalues of $\mathbf{A}$ are widely spreaded (cf. [Ang00], [AW05]). 


\subsection{The case of Donald diagrams}

Let us now consider a family of admissible (in the sense of FEM, cf. [Cia78, Ch. 2]) triangulations $\mathcal{F}=\{\mathcal{T}\}$. Then, for any $T \in \mathcal{T}$ with local vertices $z_{j} \equiv x_{i_{j}}, i_{j} \in \Lambda_{T}, j \in[1, d+1]_{\mathbb{N}}$, we define

$$
\Omega_{i_{j}, T}^{D}:=\left\{x \in T:\left(\forall k \in[1, d+1]_{\mathbb{N}} \backslash\{j\}: \lambda_{k}(x)<\lambda_{j}(x)\right)\right\},
$$

where $\lambda_{j}(x)$ is the $j$-th barycentric coordinate of $x$ w.r.t. $T$. Define for $i \in \bar{\Lambda}$ the sets

$$
\Omega_{i}^{D}:=\operatorname{int}\left(\bigcup_{T: \partial T \ni x_{i}} \overline{\Omega_{i, T}^{D}}\right) .
$$

In this way, we get a family of Donald diagrams.

Although it is possible to introduce a discretization like (11), we use the following version:

$$
a_{\mathcal{T}}\left(w_{\mathcal{T}} ; v_{\mathcal{T}}\right)=\left(\mathbf{A}\left(\cdot, w_{\mathcal{T}}\right) \nabla w_{\mathcal{T}}, \nabla v_{\mathcal{T}}\right)+b_{\mathcal{T}}\left(w_{\mathcal{T}} ; v_{\mathcal{T}}\right)+d_{\mathcal{T}}\left(w_{\mathcal{T}} ; v_{\mathcal{T}}\right)
$$

where the forms $b_{\mathcal{T}}, d_{\mathcal{T}}$ are defined analogously to (9),(10). In particular, $\gamma_{i j} \in \mathbb{R}$ is an approximation to $\left.(\nu \cdot \mathbf{b})\left(\cdot, w_{\mathcal{T}}\right)\right|_{\Gamma i j}$.

In the case of a matrix-valued diffusion coefficient, we define $\mu_{i j}$ analogously to (12) but use it only in $b_{\mathcal{T}}$ to ensure a certain stabilization. The form $a_{\mathcal{T}}^{0}$ remains as it is, i.e. $a_{\mathcal{T}}^{0}\left(w_{\mathcal{T}} ; v_{\mathcal{T}}\right):=\left(\mathbf{A}\left(\cdot, w_{\mathcal{T}}\right) \nabla w_{\mathcal{T}}, \nabla v_{\mathcal{T}}\right)$.

\section{$3 \quad$ Stability and a priori error estimates}

\subsection{The case of a linear equation with a scalar diffusion coefficient}

In this section we give a short review of some wellknown properties of the schemes (6) and (13) for the case of a linear equation with a scalar diffusion coefficient. We start with the formulation of conditions with respect to the approximations $\mu_{i j}$ and $\gamma_{i j}$.

(A2.1) $\mu_{i j}$ is an approximation of the term $m_{i j}^{-1} \int_{\Gamma_{i j}} A d s$ satisfying the following conditions:

(i) $0 \leq \mu_{i j} \leq\|A\|_{1, \infty, \Omega}$,

(ii) $\mu_{i j}=\mu_{j i}$,

(iii) $\left|\mu_{i j}-m_{i j}^{-1} \int_{\Gamma_{i j}} A d s\right| \leq C h_{T}|A|_{1, \infty, \Omega}$, where $T$ is one of the simplices having the vertices $x_{i}, x_{j}$, and $C>0$ is a constant independent of $a, h_{T}, i, j$.

(A2.2) $\gamma_{i j}$ is an approximation of the term $m_{i j}^{-1} \int_{\Gamma_{i j}} \nu \cdot \mathbf{b} d s$ satisfying the following conditions:

(i) $\left|\gamma_{i j}\right| \leq\|\mathbf{b}\|_{1, \infty, \Omega}$,

(ii) $\gamma_{i j}=-\gamma_{j i}$,

(iii) $\left|\gamma_{i j}-m_{i j}^{-1} \int_{\Gamma_{i j}}\left(\nu_{i j} \cdot \mathbf{b}\right) d s\right| \leq C h_{T}|\mathbf{b}|_{1, \infty, \Omega}$, where $T$ is one of the simplices having the vertices $x_{i}, x_{j}$, and $C>0$ is a constant independent of $\mathbf{b}, h_{T}, i, j$.

The subsequent results are extensions of the theory developed in Ang91, Ang95b, see also KA03, Ch. 6]. 
THEOREM 1 (Discrete coercivity) Let a family $\mathcal{F}=\{\mathcal{T}\}$ of triangulations be given, where in the special case of Voronoi diagrams (i.e. $\Xi=V$ ) all elements $T$ are self-centered and in the special case of Donald diagrams (i.e. $\Xi=D$ ) the family is shape-regular. Moreover, let the assumptions (A2.1), (A2.2) be satisfied. Then, for $h_{0}>0$ sufficiently small there exist two constants $\bar{a}_{0}>0$ and $\bar{a}_{1}>0$ independent of $h$ such that for all $h \in\left(0, h_{0}\right]$ and $v_{\mathcal{T}} \in V_{\mathcal{T}}$ the relation

$$
a_{\mathcal{T}}\left(v_{\mathcal{T}} ; v_{\mathcal{T}}\right) \geq \bar{a}_{0}\left|v_{\mathcal{T}}\right|_{\Xi}^{2}+\bar{a}_{1}\left\|v_{\mathcal{T}}\right\|_{\mathcal{T}}^{2}
$$

holds.

The a priori error estimate is based on this stability property and on the following consistency result.

LEMMA 1 (Discrete consistency) Let a shape-regular family $\mathcal{F}$ of triangulations $\{\mathcal{T}\}$ be given, where in the special case of Voronoi diagrams (i.e. $\Xi=V$ ) all elements $T$ are selfcentered, and let the assumptions (A2.1), (A2.2) be satisfied. Then, if $h_{0}>0$ is sufficiently small, for any element $w \in W_{2}^{2}(\Omega) \cap V$ and any element $v_{\mathcal{T}} \in V_{\mathcal{T}}$ the estimate

$$
\begin{aligned}
& \left|a_{\mathcal{T}}\left(I_{\mathcal{T}} w ; v_{\mathcal{T}}\right)-\left(-\nabla \cdot(A \nabla w)+\mathbf{b} \cdot \nabla w+c w, L_{\mathcal{T}} v_{\mathcal{T}}\right)\right| \\
\leq & C h\|w\|_{2,2, \Omega}\left[\left|v_{\mathcal{T}}\right|_{\Xi}+\left\|v_{\mathcal{T}}\right\|_{\mathcal{T}}\right]
\end{aligned}
$$

holds for all $h \in\left(0, h_{0}\right]$, where $C>0$ is a constant which does not depend on $h$.

The proof of the following theorem is a modification of the standard proof of Strang's first lemma.

THEOREM 2 (A priori error estimate) Let a shape-regular family $\mathcal{F}$ of triangulations $\{\mathcal{T}\}$ be given, where in the special case of Voronoi diagrams (i.e. $\Xi=V$ ) all elements $T$ are self-centered, let the assumptions (A2.1), (A2.2) be satisfied and suppose that the solution $u \in V$ of problem (1) additionally belongs to $W_{2}^{2}(\Omega)$.

Then, for sufficiently small $h_{0}>0$ the estimate

$$
\left\|u-u_{\mathcal{T}}\right\|_{\Xi} \leq C h\left[\|u\|_{2,2, \Omega}+|f|_{1, q, \Omega}\right]
$$

holds for all $h \in\left(0, h_{0}\right]$, where the constant $C>0$ is independent of $h$.

\subsection{The quasilinear case}

Due to the possible structural diversity of the nonlinearities in (1), in the nonlinear situation there is not such a relatively canonical theory as in the linear case.

We mention here only a few papers which are concerned with the investigation of nodecentered finite volume methods for nonlinear elliptic (or parabolic) equations and refer to the literature cited therein: [FL01, CL05], EFG06].

\section{A posteriori error estimates for nonlinear problems}

In this section we present the general approach that does not depend on the particular discretization.

The nonlinear primal problem we are interested in is given by

$$
u \in V: a(u ; v)+a_{\delta}(u ; v)=\langle f, v\rangle \quad \forall v \in V .
$$


It represents the weak formulation of the originally given (accurate) boundary-value problem for a partial differential equation in a real Hilbert space $V$, where $f$ is a linear functional on $V$ and $\langle f, v\rangle$ denotes the value of $f$ at the element $v \in V$. The forms $a: V \times V \rightarrow \mathbb{R}$ and $a_{\delta}: V \times V \rightarrow \mathbb{R}$ are linear in the second argument but may be nonlinear in the first one. In the context of the boundary-value problem (4), the left-hand side of (44) is written in (14) as the sum $a+a_{\delta}$, where $a$ stands for a certain simplified problem and $a_{\delta}$ represents a part of the equation which is to be neglected in the practical computations. That is, the discretization applies only to $a$ in (14). The goal is to estimate the influence of both neglecting $a_{\delta}$ and discretizing $a$ and $f$ with respect to a given output functional $j: V \rightarrow \mathbb{R}$.

EXAMPLE 7 Consider (1) with

$$
\mathbf{A}(x, w):=\varepsilon(x)|w|^{\gamma(x)} \mathbf{I}, \quad \mathbf{b}(x, w):=\mathbf{b}_{0}(x)|w|^{\gamma(x) / 2}, \quad c(x, w):=c_{0}(x),
$$

where $\varepsilon, c_{0}, f, \gamma: \Omega \rightarrow \mathbb{R}$ and $\mathbf{b}_{0}: \Omega \rightarrow \mathbb{R}^{d}$ are smooth functions (satisfying certain additional conditions, in particular $-1<\gamma_{-} \leq \gamma(x) \leq \gamma_{+}<\infty$ on $\Omega$ for some constants $\left.\gamma_{-}, \gamma_{+}\right)$. Then, for some constant elements $w_{0}, \gamma_{0} \in \mathbb{R}$, we can set

$$
a(w ; v):=\left(\varepsilon\left|w_{0}\right|^{\gamma_{0}} \nabla w, \nabla v\right)+\left(\left|w_{0}\right|^{\gamma_{0} / 2} \mathbf{b}_{0} \cdot \nabla w, v\right)+\left(c_{0} w, v\right),
$$

and $a_{\delta}(w ; v)$ is the canonical error term with respect to the correct weak formulation of (1)).

The directional derivatives of $a(u ; \cdot)$ and $a_{\delta}(u ; \cdot)$ in $u$ will be denoted by $a^{\prime}(u ; \cdot, \cdot)$ and $a_{\delta}^{\prime}(u ; \cdot, \cdot)$, respectively. The form

$$
a^{\prime}(u ; w, v):=\lim _{\varepsilon \rightarrow 0} \frac{1}{\varepsilon}[a(u+\varepsilon w ; v)-a(u ; v)]
$$

is linear in $w$ and $v$. The second and third directional derivatives are denoted by $a^{\prime \prime}(u ; \cdot, \cdot, \cdot)$ and $a^{\prime \prime \prime}(u ; \cdot, \cdot, \cdot, \cdot)$, respectively. In the general case of a nonlinear output functional $j$, the corresponding dual problem we will use in the analysis is the following:

$$
z \in V: a^{\prime}(u ; w, z)+a_{\delta}^{\prime}(u ; w, z)=j^{\prime}(u ; w) \quad \forall w \in V .
$$

The solution $z \in V$ of the dual problem is called influence function for the particular choice of $j$ ([A00]). The primal solution $u_{m} \in V$ and the dual solution $z_{m} \in V$ of the reduced problems are given by

$$
\begin{aligned}
u_{m} \in V: a\left(u_{m} ; v\right) & =\langle f, v\rangle \quad \forall v \in V, \\
z_{m} \in V: a^{\prime}\left(u_{m} ; w, z_{m}\right) & =j^{\prime}\left(u_{m} ; w\right) \quad \forall w \in V .
\end{aligned}
$$

These variational problems will be formulated in terms of optimization problems. The primal and dual solutions will be expressed by the variables $x:=(u, z) \in X:=V \times V$ and $x_{m}:=$ $\left(u_{m}, z_{m}\right) \in X$. In the variational space $X$, we consider the functionals

$$
\begin{aligned}
L(x) & :=L_{m}(x)+L_{\delta}(x), \\
L_{m}(x) & :=j(u)+\langle f, z\rangle-a(u ; z), \\
L_{\delta}(x) & :=-a_{\delta}(u ; z) .
\end{aligned}
$$

The derivative of $L$ applied to a test function $y=(w, v) \in X$ is

$$
L^{\prime}(x ; y)=j^{\prime}(u ; w)-a^{\prime}(u ; w, z)-a_{\delta}^{\prime}(u ; w, z)+\langle f, v\rangle-a(u ; v)-a_{\delta}(u ; v) .
$$


Obviously, the original primal and dual problems (14) and (15) and the reduced primal and dual problems (16) and (17) consist of finding the stationary points $x=(u, z)$ and $x_{m}=\left(u_{m}, z_{m}\right)$ of $L$ and $L_{m}$, respectively:

$$
\begin{aligned}
x \in X: L^{\prime}(x ; y) & =0 \quad \forall y \in X, \\
x_{m} \in X: L_{m}^{\prime}\left(x_{m} ; y\right) & =0 \quad \forall y \in X .
\end{aligned}
$$

Furthermore, the target quantities are given by evaluation of $L$ and $L_{m}$ at the following stationary points:

$$
j(u)=L(x), \quad j\left(u_{m}\right)=L_{m}\left(x_{m}\right) .
$$

In order to balance the model and discretization errors, we have to include the discretization error in the analysis. To do this, let $V_{\mathcal{T}} \subset V$ be a finite-dimensional subspace. Typically $V_{\mathcal{T}}$ is a finite element space with respect to a partition $\mathcal{T}$ of the computational domain $\Omega \subset \mathbb{R}^{d}$, $d \in\{2,3\}$, where possible homogeneous Dirichlet boundary conditions are already included in the choice of the spaces $V$ and $V_{\mathcal{T}}$. Let $a_{\mathcal{T}}: V_{\mathcal{T}} \times V_{\mathcal{T}} \rightarrow \mathbb{R}$ be a nonlinear form which is different, in general, from the simple restriction of $a$ to $V_{\mathcal{T}} \times V_{\mathcal{T}}$, and denote by $f_{\mathcal{T}}: V_{\mathcal{T}} \rightarrow \mathbb{R}$ a linear functional which not necessarily coincides with $\left.f\right|_{V_{\mathcal{T}}}$. For instance, $a_{\mathcal{T}}$ and $f_{\mathcal{T}}$ may result from the finite volume discretization of $a, f$ in (14) according to Section 2 ,

Then $u_{\mathcal{T} m} \in V_{\mathcal{T}}$ is the discrete solution of the problem

$$
u_{\mathcal{T} m} \in V_{\mathcal{T}}: a_{\mathcal{T}}\left(u_{\mathcal{T} m} ; v\right)=\left\langle f_{\mathcal{T}}, v\right\rangle \quad \forall v \in V_{\mathcal{T}}
$$

involving both types of error. The operators $L$ and $L_{m}$ are still given by (18)-(20). The difference lies in the definition of the discrete solution $x_{\mathcal{T} m}=\left(u_{\mathcal{T} m}, z_{\mathcal{T} m}\right) \in X_{\mathcal{T}}:=V_{\mathcal{T}} \times V_{\mathcal{T}}$, where now $u_{\mathcal{T} m}$ satisfies (23) and $z_{\mathcal{T} m}$ is the solution of the following dual problem:

$$
z_{\mathcal{T} m} \in V_{\mathcal{T}}: a^{\prime}\left(u_{\mathcal{T} m} ; w, z_{\mathcal{T} m}\right)=j^{\prime}\left(u_{\mathcal{T} m} ; w\right) \quad \forall w \in V_{\mathcal{T}}
$$

In such a setting, the relations $a\left(u_{\mathcal{T} m} ; v\right)=\langle f, v\rangle$ and $L_{m}^{\prime}\left(x_{\mathcal{T} m} ; y\right)=0$ are no longer valid for all $v \in V_{\mathcal{T}}$ resp. $y \in X_{\mathcal{T}}$.

The target quantities are given by the evaluation of $L$ and $L_{\mathcal{T} m}$, where

$$
L_{\mathcal{T} m}(x):=j(u)+\left\langle f_{\mathcal{T}}, z\right\rangle-a_{\mathcal{T}}(u ; z),
$$

at the following stationary points:

$$
j(u)=L(x), \quad j\left(u_{m}\right)=L_{\mathcal{T} m}\left(x_{m}\right) .
$$

For the formulation of the error representation, we use the following notation for the primal and dual residual with respect to the reduced model and for test functions $(w, v) \in X$ :

$$
\begin{aligned}
\varrho\left(u_{\mathcal{T} m} ; v\right) & :=\langle f, v\rangle-a\left(u_{\mathcal{T} m} ; v\right), \\
\varrho^{*}\left(u_{\mathcal{T} m} ; z_{\mathcal{T} m}, w\right) & :=j^{\prime}\left(u_{\mathcal{T} m} ; w\right)-a^{\prime}\left(u_{\mathcal{T} m} ; w, z_{\mathcal{T} m}\right) .
\end{aligned}
$$


THEOREM 3 If $a(u ; \cdot), a_{\delta}(u ; \cdot)$ and the functional $j(u)$ are sufficiently differentiable with respect to $u$, then we have

$$
\begin{aligned}
j(u)-j\left(u_{\mathcal{T} m}\right)= & -a_{\delta}\left(u_{\mathcal{T} m} ; z_{\mathcal{T} m}\right) \\
& +\left\langle f, z_{\mathcal{T} m}\right\rangle-\left\langle f_{\mathcal{T}}, z_{\mathcal{T} m}\right\rangle-a\left(u_{\mathcal{T} m} ; z_{\mathcal{T} m}\right)+a_{\mathcal{T}}\left(u_{\mathcal{T} m} ; z_{\mathcal{T} m}\right) \\
& +\frac{1}{2}\left[\varrho\left(u_{\mathcal{T} m} ; z-i_{\mathcal{T}} z\right)+\varrho^{*}\left(u_{\mathcal{T} m} ; z_{\mathcal{T} m}, u-i_{\mathcal{T}} u\right)\right] \\
& -\frac{1}{2}\left[a_{\delta}\left(u_{\mathcal{T} m} ; e_{z}\right)+a_{\delta}^{\prime}\left(u_{\mathcal{T} m} ; e_{u}, z_{\mathcal{T} m}\right)\right] \\
& -\frac{1}{2} \varrho\left(u_{\mathcal{T} m} ; z_{\mathcal{T} m}-i_{\mathcal{T}} z\right)-\frac{1}{2} R
\end{aligned}
$$

where $e:=\left(e_{u}, e_{z}\right):=\left(u-u_{\mathcal{T} m}, z-z_{\mathcal{T} m}\right), i_{\mathcal{T}}: V \rightarrow V_{\mathcal{T}}$ is an interpolation operator, and the remainder $R$ is given by

$$
R:=\int_{0}^{1} \sigma(1-\sigma) L^{\prime \prime \prime}\left(x_{\mathcal{T} m}+\sigma e ; e, e, e\right) d \sigma .
$$

Proof: By (26)),

$$
\begin{aligned}
j(u)-j\left(u_{\mathcal{T} m}\right)= & L(x)-L_{\mathcal{T} m}\left(x_{\mathcal{T} m}\right) \\
= & L(x)-L_{m}\left(x_{\mathcal{T} m}\right)+L_{m}\left(x_{\mathcal{T} m}\right)-L_{\mathcal{T} m}\left(x_{\mathcal{T} m}\right) \\
= & L(x)-L_{m}\left(x_{\mathcal{T} m}\right) \\
& +\left\langle f, z_{\mathcal{T} m}\right\rangle-\left\langle f_{\mathcal{T}}, z_{\mathcal{T} m}\right\rangle-a\left(u_{\mathcal{T} m} ; z_{\mathcal{T} m}\right)+a_{\mathcal{T}}\left(u_{\mathcal{T} m} ; z_{\mathcal{T} m}\right),
\end{aligned}
$$

where the last step is a consequence of the definitions (20), (25)).

The first difference can be estimated as in the proof of [BE03, Thm. 2.1]:

$$
\begin{aligned}
L(x)-L_{m}\left(x_{\mathcal{T} m}\right) & =L(x)-L\left(x_{\mathcal{T} m}\right)+L_{\delta}\left(x_{\mathcal{T} m}\right) \\
& =\int_{0}^{1} L^{\prime}\left(x_{\mathcal{T} m}+\sigma\left(x-x_{\mathcal{T} m}\right) ; x-x_{\mathcal{T} m}\right) d \sigma+L_{\delta}\left(x_{\mathcal{T} m}\right) \\
& =\frac{1}{2}\left[L^{\prime}\left(x_{\mathcal{T} m} ; e\right)+L^{\prime}(x ; e)-R\right]-a_{\delta}\left(u_{\mathcal{T} m} ; z_{\mathcal{T} m}\right)
\end{aligned}
$$

with the above given remainder $R$ of the trapezoidal rule. Since $L^{\prime}(x ; e)=0$ by (21), we get

$$
L(x)-L_{m}\left(x_{\mathcal{T} m}\right)=-a_{\delta}\left(u_{\mathcal{T} m} ; z_{\mathcal{T} m}\right)+\frac{1}{2}\left[L^{\prime}\left(x_{\mathcal{T} m} ; e\right)-R\right] .
$$

Furthermore,

$$
\begin{aligned}
L^{\prime}\left(x_{\mathcal{T} m} ; e\right)= & j^{\prime}\left(u_{\mathcal{T} m} ; e_{u}\right)-a^{\prime}\left(u_{\mathcal{T} m} ; e_{u}, z_{\mathcal{T} m}\right)-a_{\delta}^{\prime}\left(u_{\mathcal{T} m} ; e_{u}, z_{\mathcal{T} m}\right) \\
& +\left\langle f, e_{z}\right\rangle-a\left(u_{\mathcal{T} m} ; e_{z}\right)-a_{\delta}\left(u_{\mathcal{T} m} ; e_{z}\right) \\
= & \varrho^{*}\left(u_{\mathcal{T} m} ; z_{\mathcal{T} m}, e_{u}\right)-a_{\delta}^{\prime}\left(u_{\mathcal{T} m} ; e_{u}, z_{\mathcal{T} m}\right)+\varrho\left(u_{\mathcal{T} m} ; e_{z}\right)-a_{\delta}\left(u_{\mathcal{T} m} ; e_{z}\right) .
\end{aligned}
$$

Since the Galerkin orthogonality is violated, in general, we cannot use the standard argument

$$
0=\varrho\left(u_{\mathcal{T} m} ; z_{\mathcal{T} m}\right)=\varrho\left(u_{\mathcal{T} m} ; i_{\mathcal{T} z}\right)
$$


to replace $z_{\mathcal{T} m}$ by $i_{\mathcal{T}} z$ in the third term. Here we can only make use of an analogous property of the dual problem (24), i.e.

$$
0=\varrho^{*}\left(u_{\mathcal{T} m} ; z_{\mathcal{T} m}, u_{\mathcal{T} m}\right)=\varrho^{*}\left(u_{\mathcal{T} m} ; z_{\mathcal{T} m}, i_{\mathcal{T}} u\right) .
$$

(Of course, if the dual problem is approximated by a finite volume method, too, then we have to argue as for the primal problem.) Thus we arrive at

$$
\begin{aligned}
L^{\prime}\left(x_{\mathcal{T} m} ; e\right)= & \varrho^{*}\left(u_{\mathcal{T} m} ; z_{\mathcal{T} m}, u-i_{\mathcal{T} u}\right)+\varrho\left(u_{\mathcal{T} m} ; z-i_{\mathcal{T}} z\right) \\
& -\varrho\left(u_{\mathcal{T} m} ; z_{\mathcal{T} m}-i_{\mathcal{T}} z\right)-a_{\delta}\left(u_{\mathcal{T} m} ; e_{z}\right)-a_{\delta}^{\prime}\left(u_{\mathcal{T} m} ; e_{u}, z_{\mathcal{T} m}\right) .
\end{aligned}
$$

This gives the assertion.

In order to use numerically the error representation derived in Theorem 3, we have to approximate various terms. In particular, we will neglect the higher-order terms in $e$, namely the remainder $R$ and the terms $a_{\delta}\left(u_{\mathcal{T} m} ; e_{z}\right), a_{\delta}^{\prime}\left(u_{\mathcal{T} m} ; e_{u}, z_{\mathcal{T} m}\right)$, cf. the related discussion in BE03. Furthermore, we have to approximate the interpolation errors $u-i_{\mathcal{T}} u$ and $z-i_{\mathcal{T}} z$. An efficient possibility for doing this is the recovery process of the computed quantities by patchwise higher-order interpolation expressed via the operator $i_{\mathcal{T}}^{+}: V_{\mathcal{T}} \rightarrow V_{\mathcal{T}}^{+}$formally, where $V_{\mathcal{T}}^{+}$is a richer discrete space than $V_{\mathcal{T}}$ (see [BR01, Sect. 5], [Ran05, Sect. 3.2]). For instance, in the case of triangles $(d=2)$ or tetrahedra $(d=3)$ and when $V_{\mathcal{T}}$ consists of piecewise linear elements, quadratic interpolation may be used. For quadrilaterals and piecewise $d$ linear elements, the interpolation can be done on $d$-quadratic elements. In order to preserve a sufficient high accuracy of the interpolation procedure, special care on elements with hanging nodes is required.

The interpolation errors will be numerically approximated by

$$
\begin{aligned}
z-i_{\mathcal{T}} z & \approx i_{\mathcal{T}}^{+} z_{\mathcal{T} m}-z_{\mathcal{T} m}, \\
u-i_{\mathcal{T}} u & \approx i_{\mathcal{T}}^{+} u_{\mathcal{T} m}-u_{\mathcal{T} m} .
\end{aligned}
$$

Without the modeling error and in the case of conforming methods, this approximation is usually observed to be accurate enough.

Taking into account that the residual $\varrho^{*}\left(u_{\mathcal{T} m} ; z_{\mathcal{T} m}, v\right)$ vanishes with respect to a discrete test function $v \in V_{\mathcal{T}}$, we obtain from Theorem 3 the following approximate estimator consisting of three indicators:

$$
\begin{aligned}
j(u)-j\left(u_{\mathcal{T} m}\right) & \approx \eta_{\mathcal{T}}+\eta_{m}+\eta_{n c} \\
\eta_{\mathcal{T}} & :=\frac{1}{2}\left[\varrho\left(u_{\mathcal{T} m} ; i_{\mathcal{T}}^{+} z_{\mathcal{T} m}-z_{\mathcal{T} m}\right)+\varrho^{*}\left(u_{\mathcal{T} m} ; z_{\mathcal{T} m}, i_{\mathcal{T}}^{+} u_{\mathcal{T} m}\right)\right] \\
\eta_{m} & :=-a_{\delta}\left(u_{\mathcal{T} m} ; z_{\mathcal{T} m}\right), \\
\eta_{n c} & :=\left\langle f, z_{\mathcal{T} m}\right\rangle-\left\langle f_{\mathcal{T}}, z_{\mathcal{T} m}\right\rangle-a\left(u_{\mathcal{T} m} ; z_{\mathcal{T} m}\right)+a_{\mathcal{T}}\left(u_{\mathcal{T} m} ; z_{\mathcal{T} m}\right) .
\end{aligned}
$$

The indicator $\eta_{\mathcal{T}}$ of the approximate estimator can be considered as the conforming contribution of the discretization, and the indicator $\eta_{m}$ measures the influence of the model. For complex models, the evaluation of $\eta_{m}$ may be expensive. Often in practice the decomposition $a+a_{\delta}$ is changed successively in such a way that portions of $a_{\delta}$ are (locally) shifted to $a$. The indicator $\eta_{n c}$ results from the nonconformity of the discretization method caused by the violation of the Galerkin orthogonality. The practical treatment of $\eta_{n c}$ will be discussed in Section 5 . 
REMARK 3 According to (28), there are two ways for the treatment of the term

$$
\varrho\left(u_{\mathcal{T} m} ; z-i_{\mathcal{T}} z\right)-\varrho\left(u_{\mathcal{T} m} ; z_{\mathcal{T} m}-i_{\mathcal{T}} z\right)
$$

occuring in Theorem 3. Either we write it as $\varrho\left(u_{\mathcal{T} m} ; z-z_{\mathcal{T} m}\right)$ (i.e. we reverse the splitting used in (27)) and replace then $z$ by $i_{\mathcal{T}}^{+} z_{\mathcal{T} m}$, or we replace $z-i_{\mathcal{T}} z$ by $i_{\mathcal{T}}^{+} z_{\mathcal{T} m}-z_{\mathcal{T} m}$ and $i_{\mathcal{T}} z$ by $z_{\mathcal{T} m}$. In both cases, we arrive at the same result:

$$
\varrho\left(u_{\mathcal{T} m} ; z-i_{\mathcal{T}} z\right)-\varrho\left(u_{\mathcal{T} m} ; z_{\mathcal{T} m}-i_{\mathcal{T}} z\right) \approx \varrho\left(u_{\mathcal{T} m} ; i_{\mathcal{T}}^{+} z_{\mathcal{T} m}-z_{\mathcal{T} m}\right) .
$$

In order to use the information (29) for changing locally the model or the discretization parameters (e.g. the mesh size), we have to localize the indicators. After that, an adaptive process has to be designed in order to balance the error sources.

Regarding the localization of $\eta_{\mathcal{T}}$ and $\eta_{m}$, so here there are no new aspects. We refer, for instance, to BE03].

\section{Application to the finite volume method}

In the papers Ang91, Ang92, an extension of Babuška\&Rheinboldt's a posteriori error estimates for finite element methods ([BR78]) to finite volume methods for linear diffusionconvection eqations has been proposed. In a subsequent paper (Ang95a), for a singularly perturbed model problem a modification was introduced with the aim to get two-sided bounds of the error such that the constants occuring in these bounds are independent of the perturbation parameter. In AKT98 and Thi99, residual type error estimates for finite volume discretizations of more complicated problems in two and three space dimensions have been presented. A rather general framework for the a posteriori estimation in various finite volume methods can be found in Voh08, however this paper is restricted to linear problems and estimates w.r.t. the energy norm. In Ang10, dual-weighted residual error estimators for finite volume discretizations of linear diffusion-convection eqations have been described. Here we apply the results of the previous section to the nonlinear diffusion-convection problem. As a result, we get a posteriori estimates for errors of functionals depending nonlinearly on the solution and for possible modeling errors.

Interpreting $a_{\mathcal{T}}$ and $f_{\mathcal{T}}$ as the finite volume discretizations (6) of the forms $a$ and $f$ in (14), we first observe that the the estimators $\eta_{\mathcal{T}}$ and $\eta_{m}$ depend only on the computed discrete solution but not directly on the structure of $a_{\mathcal{T}}$ and $f_{\mathcal{T}}$. Therefore, these estimators can be treated as in the (conforming) finite element case and we concentrate on the estimator $\eta_{n c}$. To simplify the presentation, we will write $x_{\mathcal{T}}=\left(u_{\mathcal{T}}, z_{\mathcal{T}}\right)$ instead of $x_{\mathcal{T} m}=\left(u_{\mathcal{T} m}, z_{\mathcal{T} m}\right)$.

Then, by definition, we have that

$$
\begin{aligned}
\left\langle f, z_{\mathcal{T}}\right\rangle-\left\langle f_{\mathcal{T}}, z_{\mathcal{T}}\right\rangle & =\sum_{T \in \mathcal{T}}\left\{\left(f, z_{\mathcal{T}}\right)_{T}-\left(f, z_{\mathcal{T}}\right)_{l, T}\right\} \\
& :=\sum_{T \in \mathcal{T}}\left\{\left(f, z_{\mathcal{T}}\right)_{T}-\sum_{i \in \Lambda_{T}} f_{i} z_{\mathcal{T} i} m_{i}^{T}\right\},
\end{aligned}
$$

where $\left(f, z_{\mathcal{T}}\right)_{T}:=\int_{T} f z_{\mathcal{T}} d x$ and $m_{i}^{T}:=\operatorname{meas}_{d}\left(\Omega_{i} \cap T\right)$. Analogously, with

$$
\begin{aligned}
& a_{\mathcal{T}, T}\left(u_{\mathcal{T}} ; z_{\mathcal{T}}\right) \\
:= & \sum_{i \in \Lambda} z_{\mathcal{T} i}\left\{\sum_{j \in \Lambda_{T} \backslash\{i\}}\left\{\mu_{i j} \frac{u_{\mathcal{T} i}-u_{\mathcal{T} j}}{d_{i j}}-\gamma_{i j}\left(1-r_{i j}\right)\left(u_{\mathcal{T} i}-u_{\mathcal{T} j}\right)\right\} m_{i j}^{T}+c_{i} u_{\mathcal{T} i} m_{i}^{T}\right\}
\end{aligned}
$$


and $a_{T}\left(u_{\mathcal{T}} ; z_{\mathcal{T}}\right)$ resulting from the restriction of all integrals occuring in the expression for $a\left(u_{\mathcal{T}} ; z_{\mathcal{T}}\right)$ to the domain of integration $T$, we have that

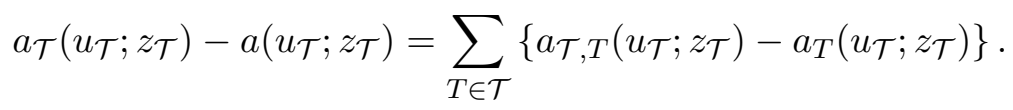

Putting (30) and (31) together, we conclude that

$$
\eta_{n c}=\sum_{T \in \mathcal{T}}\left\{\left(f, z_{\mathcal{T}}\right)_{T}-\sum_{i \in \Lambda_{T}} f_{i} z_{\mathcal{T} i} m_{i}^{T}\right\}+\sum_{T \in \mathcal{T}}\left\{a_{\mathcal{T}, T}\left(u_{\mathcal{T}} ; z_{\mathcal{T}}\right)-a_{T}\left(u_{\mathcal{T}} ; z_{\mathcal{T}}\right)\right\}
$$

This is the starting point for the practical, localized computation.

\section{Analysis of the nonconformity estimator}

In this section we show for the case of a scalar diffusion coefficient $\mathbf{A}=A \mathbf{I}$ with $A: \Omega \times \mathbb{R} \rightarrow$ $\mathbb{R}$ that $\eta_{n c}$ is order-consistent with the a priori error estimate (Theorem 2). A precise formulation of this property is given at the end of the section.

Using (30), (31), we get the following decomposition:

$$
\begin{aligned}
\eta_{n c}= & \sum_{i \in \bar{\Lambda}} \int_{\Omega_{i}}\left[f z_{\mathcal{T}}-f_{i} z_{\mathcal{T}_{i}}\right] d x \\
& +\sum_{i \in \Lambda} z_{\mathcal{T} i} \sum_{j \in \Lambda_{i}} \mu_{i j}\left(u_{\mathcal{T} i}-u_{\mathcal{T} j}\right) \frac{m_{i j}}{d_{i j}}-\left(A \nabla u_{\mathcal{T}}, \nabla z_{\mathcal{T}}\right) \\
& +\sum_{i \in \Lambda} \sum_{j \in \Lambda_{i}}\left(1-r_{i j}\right) \gamma_{i j}\left(u_{\mathcal{T} j}-u_{\mathcal{T} i}\right) z_{\mathcal{T} i} m_{i j}-\left(\mathbf{b} \cdot \nabla u_{\mathcal{T}}, z_{\mathcal{T}}\right) \\
& +\sum_{i \in \bar{\Lambda}} \int_{\Omega_{i}}\left[c_{i} u_{\mathcal{T} i} z_{\mathcal{T} i}-c u_{\mathcal{T}} z_{\mathcal{T}}\right] d x \\
= & \sum_{i \in \bar{\Lambda}}\left\{\int_{\Omega_{i}} f\left(z_{\mathcal{T}}-z_{\mathcal{T} i}\right) d x+\int_{\Omega_{i}}\left(f-f_{i}\right) z_{\mathcal{T} i} d x\right\} \\
& +\sum_{i \in \Lambda} z_{\mathcal{T} i} \sum_{j \in \Lambda_{i}} \mu_{i j}\left(u_{\mathcal{T} i}-u_{\mathcal{T} j}\right) \frac{m_{i j}}{d_{i j}}-\left(A \nabla u_{\mathcal{T}}, \nabla z_{\mathcal{T}}\right) \\
& +\sum_{i \in \Lambda}\left\{\sum_{j \in \Lambda_{i}}\left(1-r_{i j}\right) \gamma_{i j}\left(u_{\mathcal{T} j}-u_{\mathcal{T}_{i}}\right) z_{\mathcal{T} i} m_{i j}-\int_{\Omega_{i}}\left(\mathbf{b} \cdot \nabla u_{\mathcal{T}}\right) z_{\mathcal{T} i} d x\right\} \\
& -\sum_{i \in \bar{\Lambda}} \int_{\Omega_{i}}\left(\mathbf{b} \cdot \nabla u_{\mathcal{T}}\right)\left(z_{\mathcal{T}}-z_{\mathcal{T}_{i}}\right) d x \\
& +\sum_{i \in \bar{\Lambda}}\left\{\int_{\Omega_{i}}\left[c_{i} u_{\mathcal{T} i}-c u_{\mathcal{T}}\right] z_{\mathcal{T} i} d x-\int_{\Omega_{i}} c u_{\mathcal{T}}\left(z \mathcal{T}-z_{\mathcal{T} i}\right) d x\right\} \\
= & \delta_{0}+\delta_{1}+\delta_{2}+\delta_{3}
\end{aligned}
$$


with

$$
\begin{aligned}
\delta_{0} & :=\sum_{i \in \Lambda} z_{\mathcal{T} i} \sum_{j \in \Lambda_{i}} \mu_{i j}\left(u_{\mathcal{T} i}-u_{\mathcal{T} j}\right) \frac{m_{i j}}{d_{i j}}-\left(A \nabla u_{\mathcal{T}}, \nabla z_{\mathcal{T}}\right), \\
\delta_{1} & :=\sum_{i \in \Lambda} \int_{\Omega_{i}}\left[f-\mathbf{b} \cdot \nabla u_{\mathcal{T}}-c u_{\mathcal{T}}\right]\left(z_{\mathcal{T}}-z_{\mathcal{T} i}\right) d x, \\
\delta_{2} & :=\sum_{i \in \Lambda} z_{\mathcal{T} i}\left\{\int_{\Omega_{i}}\left[f-f_{i}+(\nabla \cdot \mathbf{b}-c) u_{\mathcal{T}}+c_{i} u_{\mathcal{T} i}\right] d x-\sum_{j \in \Lambda_{i}} u_{\mathcal{T} i} \gamma_{i j} m_{i j}\right\}, \\
\delta_{3} & :=\sum_{i \in \Lambda} \sum_{j \in \Lambda_{i}} \int_{\Gamma_{i j}}\left[\left(r_{i j} u_{\mathcal{T} i}+\left(1-r_{i j}\right) u_{\mathcal{T}_{j}}\right) \gamma_{i j}-\left(\nu_{i j} \cdot \mathbf{b}\right) u_{\mathcal{T}}\right] z_{\mathcal{T} i} d s .
\end{aligned}
$$

Here we have used that $\mathbf{b} \cdot \nabla u_{\mathcal{T}}=\nabla \cdot\left(\mathbf{b} u_{\mathcal{T}}\right)-(\nabla \cdot \mathbf{b}) u_{\mathcal{T}}$.

In the case of Donald diagrams, $\delta_{0}=0$.

In order to treat $\delta_{0}$ in the case of Voronoi diagrams, we introduce a piecewise constant (w.r.t. $\mathcal{T}$ ) approximation $A_{\mathcal{T}}$ to $A$ by $\left.A_{\mathcal{T}}\right|_{T}:=\frac{1}{\operatorname{meas}_{d}(T)} \int_{T} A d x, T \in \mathcal{T}$. Then we can write

$$
\begin{aligned}
\delta_{0}= & \sum_{i \in \Lambda} z_{\mathcal{T} i} \sum_{j \in \Lambda_{i}}\left(\mu_{i j}-\frac{1}{m_{i j}} \int_{\Gamma_{i j}} A_{\mathcal{T}} d s\right)\left(u_{\mathcal{T} i}-u_{\mathcal{T} j}\right) \frac{m_{i j}}{d_{i j}} \\
& +\sum_{i \in \Lambda} z_{\mathcal{T} i} \sum_{j \in \Lambda_{i}}\left(\int_{\Gamma_{i j}} A_{\mathcal{T}} d s\right) \frac{u_{\mathcal{T} i}-u_{\mathcal{T} j}}{d_{i j}}-\left(A \nabla u_{\mathcal{T}}, \nabla z_{\mathcal{T}}\right) .
\end{aligned}
$$

It is wellknown that, for arbitrary $u_{\mathcal{T}}, z_{\mathcal{T}} \in V_{\mathcal{T}}$,

$$
\sum_{i \in \Lambda} z_{\mathcal{T} i} \sum_{j \in \Lambda_{i}}\left(\int_{\Gamma_{i j}} A_{\mathcal{T}} d s\right) \frac{u_{\mathcal{T} i}-u_{\mathcal{T} j}}{d_{i j}}=\left(A_{\mathcal{T}} \nabla u_{\mathcal{T}}, \nabla z_{\mathcal{T}}\right)
$$

Hence

$$
\delta_{0}=\sum_{i \in \Lambda} z_{\mathcal{T}} \sum_{j \in \Lambda_{i}}\left(\mu_{i j}-\frac{1}{m_{i j}} \int_{\Gamma_{i j}} A_{\mathcal{T}} d s\right)\left(u_{\mathcal{T} i}-u_{\mathcal{T} j}\right) \frac{m_{i j}}{d_{i j}}+\left(\left(A_{\mathcal{T}}-A\right) \nabla u_{\mathcal{T}}, \nabla z_{\mathcal{T}}\right) .
$$

Since both $\nabla u_{\mathcal{T}}, \nabla z_{\mathcal{T}}$ are constant on every element $T \in \mathcal{T}$, the second term vanishes. By a symmetry argument, we arrive at

$$
\delta_{0}=\frac{1}{2} \sum_{i \in \Lambda} \sum_{j \in \Lambda_{i}}\left(\mu_{i j}-\frac{1}{m_{i j}} \int_{\Gamma_{i j}} A_{\mathcal{T}} d s\right)\left(u_{\mathcal{T} i}-u_{\mathcal{T} j}\right)\left(z_{\mathcal{T} i}-z_{\mathcal{T} j}\right) \frac{m_{i j}}{d_{i j}} .
$$

Now the Cauchy-Schwarz inequality implies

$$
\begin{aligned}
\left|\delta_{0}\right| \leq & \frac{1}{2}\left\{\sum_{i \in \Lambda} \sum_{j \in \Lambda_{i}}\left(\mu_{i j}-\frac{1}{m_{i j}} \int_{\Gamma_{i j}} A_{\mathcal{T}} d s\right)^{2}\left(u_{\mathcal{T} i}-u_{\mathcal{T} j}\right)^{2} \frac{m_{i j}}{d_{i j}}\right\}^{1 / 2} \\
& \times\left\{\sum_{i \in \Lambda} \sum_{j \in \Lambda_{i}}\left(z_{\mathcal{T} i}-z_{\mathcal{T} j}\right)^{2} \frac{m_{i j}}{d_{i j}}\right\}^{1 / 2} .
\end{aligned}
$$




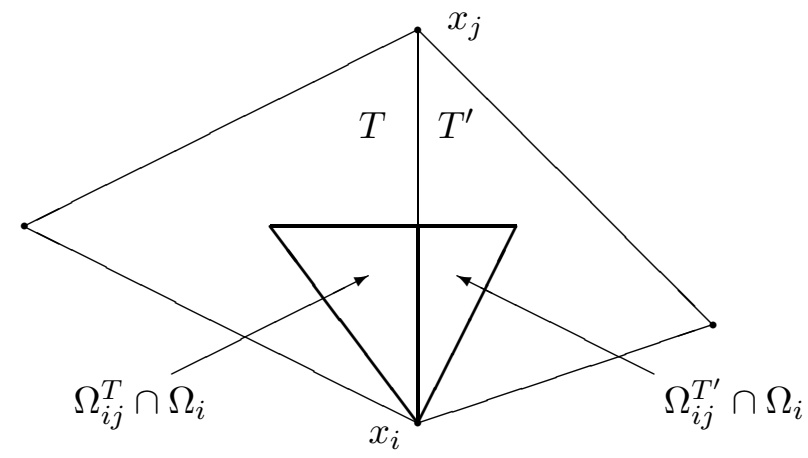

Figure 2: The auxiliary simplices in the case $d=2$ for the Voronoi diagram

The last factor can be bounded by $C_{1}\left|z_{\mathcal{T}}\right|_{1,2, \Omega}$, therefore we get

$$
\left|\delta_{0}\right| \leq C_{1} \eta_{0}\left|z_{\mathcal{T}}\right|_{1,2, \Omega}
$$

where

$$
\eta_{0}^{2}:=\sum_{i \in \Lambda} \eta_{0 i}^{2} \quad \text { with } \quad \eta_{0 i}^{2}:=\frac{1}{4} \sum_{j \in \Lambda_{i}}\left(\mu_{i j}-\frac{1}{m_{i j}} \int_{\Gamma_{i j}} A_{\mathcal{T}} d s\right)^{2}\left(u_{\mathcal{T} i}-u_{\mathcal{T} j}\right)^{2} \frac{m_{i j}}{d_{i j}}
$$

Setting $g:=f-\mathbf{b} \cdot \nabla u_{\mathcal{T}}-c u_{\mathcal{T}}$ and $\delta_{1 i}:=\int_{\Omega_{i}} g\left(z_{\mathcal{T}}-z_{\mathcal{T} i}\right) d x$, we can write (cf. Figure 2 for the case $d=2)$ :

$$
\delta_{1 i}=\sum_{j \in \Lambda_{i}} \sum_{T \in \mathcal{T}: m_{i j}^{T}>0} \int_{\Omega_{i j}^{T} \cap \Omega_{i}} g\left(z_{\mathcal{T}}-z_{\mathcal{T} i}\right) d x
$$

On each simplex $T$, it holds

$$
z_{\mathcal{T}}=z_{\mathcal{T} i}+\nabla z_{\mathcal{T}} \cdot\left(x-x_{i}\right)
$$

where $\nabla z_{\mathcal{T}}$ is constant on $\Omega_{i j}^{T}$. 
It follows that

$$
\begin{aligned}
\delta_{1 i}= & \sum_{j \in \Lambda_{i}} \sum_{T \in \mathcal{T}: m_{i j}^{T}>0} \int_{\Omega_{i j}^{T} \cap \Omega_{i}} g \nabla z_{\mathcal{T}} \cdot\left(x-x_{i}\right) d x \\
\leq & \sum_{j \in \Lambda_{i}} \sum_{T \in \mathcal{T}: m_{i j}^{T}>0} \int_{\Omega_{i j}^{T} \cap \Omega_{i}}|g|\left\|\nabla z_{\mathcal{T}}\right\|\left\|x-x_{i}\right\| d x \\
\leq & \left\{\sum_{j \in \Lambda_{i}} \sum_{T \in \mathcal{T}: m_{i j}^{T}>0} \int_{\Omega_{i j}^{T} \cap \Omega_{i}}|g|^{2}\left\|x-x_{i}\right\|^{2} d x\right\}^{1 / 2} \\
& \times\left\{\sum_{j \in \Lambda_{i}} \sum_{T \in \mathcal{T}: m_{i j}^{T}>0} \int_{\Omega_{i j}^{T} \cap \Omega_{i}}\left\|\nabla z_{\mathcal{T}}\right\|^{2} d x\right\}^{1 / 2} \\
\leq & \left\{\sum_{j \in \Lambda_{i}} \sum_{T \in \mathcal{T}: m_{i j}^{T}>0} h_{T}^{2} \int_{\Omega_{i j}^{T} \cap \Omega_{i}}|g|^{2} d x\right\}^{1 / 2}\left|z_{\mathcal{T}}\right|_{1,2, \Omega_{i}} \\
\leq & \eta_{1 i}\left|z_{\mathcal{T}}\right| 1,2, \Omega_{i},
\end{aligned}
$$

where

$$
\eta_{1}^{2}:=\sum_{i \in \bar{\Lambda}} \eta_{1 i}^{2} \quad \text { with } \quad \eta_{1 i}^{2}:=\sum_{j \in \Lambda_{i}} \sum_{T \in \mathcal{T}: m_{i j}^{T}>0} h_{T}^{2} \int_{\Omega_{i j}^{T} \cap \Omega_{i}} g^{2} d x .
$$

Thus we arrive at

$$
\delta_{1} \leq \eta_{1}\left|z_{\mathcal{T}}\right|_{1,2, \Omega}
$$

For the third term $\delta_{2}$, with

$$
\theta_{i}:=\int_{\Omega_{i}}\left[f-f_{i}+(\nabla \cdot \mathbf{b}-c) u_{\mathcal{T}}+c_{i} u_{\mathcal{T} i}\right] d x-\sum_{j \in \Lambda_{i}} u_{\mathcal{T} i} \gamma_{i j} m_{i j}
$$

we have

$$
\delta_{2}=\sum_{i \in \Lambda} z_{\mathcal{T} i} \theta_{i}
$$

Because of

$$
z_{\mathcal{T} i} \theta_{i} \leq \eta_{2 i}\left|z_{\mathcal{T}}\right| \sqrt{m_{i}}
$$

where $\eta_{2 i}:=\left|\theta_{i}\right| / \sqrt{m_{i}}$, it follows with $\eta_{2}^{2}:=\sum_{i \in \Lambda} \eta_{2 i}^{2}$ that

$$
\delta_{2} \leq \eta_{2}\left\|z_{\mathcal{T}}\right\|_{\mathcal{T}}
$$

In view of the equivalence of the $L_{2}$-norm and the lumped $L_{2}$-norm on $V_{\mathcal{T}}$, we obtain

$$
\delta_{2} \leq C_{2} \eta_{2}\left\|z_{\mathcal{T}}\right\|_{0,2, \Omega} .
$$

For the remaining term $\delta_{3}$ we have, by a symmetry argument, that

$$
\delta_{3}=\sum_{i \in \Lambda} \delta_{3 i},
$$


where

$$
\delta_{3 i}:=\frac{1}{2} \sum_{j \in \Lambda_{i}} \int_{\Gamma_{i j}} \zeta_{\mathcal{T} i j}\left(z_{\mathcal{T} i}-z_{\mathcal{T} j}\right) d s
$$

with

$$
\zeta_{\mathcal{T} i j}:=\left[r_{i j} u_{\mathcal{T} i}+\left(1-r_{i j}\right) u_{\mathcal{T} j}\right] \gamma_{i j}-\left(\nu_{i j} \cdot \mathbf{b}\right) u_{\mathcal{T}} .
$$

In view of $z_{\mathcal{T} i}-z_{\mathcal{T} j}=d_{i j}\left(\nu_{i j} \cdot \nabla z_{\mathcal{T}}\right)$ on $\Omega_{i j}^{T}$ we get

$$
\delta_{3 i}=\frac{1}{2} \sum_{j \in \Lambda_{i}} d_{i j} \sum_{T \in \mathcal{T}: m_{i j}^{T}>0} \int_{\Gamma_{i j}^{T}} \zeta_{\mathcal{T} i j}\left(\nu_{i j} \cdot \nabla z_{\mathcal{T}}\right) d s .
$$

It follows (remember that $\nu_{i j} \cdot \nabla z_{\mathcal{T}}$ is constant on $\Gamma_{i j}^{T}$ and $\nabla z_{\mathcal{T}}$ is constant on $\Omega_{i j}^{T} \cap \Omega_{i}$ )

$$
\begin{aligned}
\delta_{3 i} & \leq \frac{1}{2} \sum_{j \in \Lambda_{i}} d_{i j} \sum_{T \in \mathcal{T}: m_{i j}^{T}>0}\left|\int_{\Gamma_{i j}^{T}} \zeta_{\mathcal{T} i j} d s\right|\left\|\nabla z_{\mathcal{T}}\right\| \\
& =\frac{1}{2} \sum_{j \in \Lambda_{i}} \sum_{T \in \mathcal{T}: m_{i j}^{T}>0} \frac{d_{i j}}{\sqrt{\operatorname{meas}_{d}\left(\Omega_{i j}^{T} \cap \Omega_{i}\right)}}\left|\int_{\Gamma_{i j}^{T}} \zeta_{\mathcal{T} i j} d s\right|\left\|\nabla z_{\mathcal{T}}\right\| \sqrt{\operatorname{meas}_{d}\left(\Omega_{i j}^{T} \cap \Omega_{i}\right)} .
\end{aligned}
$$

By Cauchy's inequality, we have

$$
\begin{aligned}
\delta_{3 i} & \leq \frac{1}{2}\left\{\sum_{j \in \Lambda_{i}} \sum_{T \in \mathcal{T}: m_{i j}^{T}>0} \frac{d_{i j}^{2}}{\operatorname{meas}_{d}\left(\Omega_{i j}^{T} \cap \Omega_{i}\right)}\left(\int_{\Gamma_{i j}^{T}} \zeta_{\mathcal{T} i j} d s\right)^{2}\right\}^{1 / 2}\left|z_{\mathcal{T}}\right|_{1,2, \Omega_{i}} \\
& \leq \eta_{3 i}\left|z_{\mathcal{T}}\right|_{1,2, \Omega_{i}},
\end{aligned}
$$

where

$$
\begin{aligned}
\eta_{3 i}^{2} & :=\frac{1}{4} \sum_{j \in \Lambda_{i}} \sum_{T \in \mathcal{T}: m_{i j}^{T}>0} \frac{d_{i j}^{2}}{\operatorname{meas}_{d}\left(\Omega_{i j}^{T} \cap \Omega_{i}\right)}\left(\int_{\Gamma_{i j}^{T}} \zeta_{\mathcal{T} i j} d s\right)^{2} \\
& =\frac{d}{4} \sum_{j \in \Lambda_{i}} \sum_{T \in \mathcal{T}: m_{i j}^{T}>0} \frac{d_{i j}}{m_{i j}^{T}}\left(\int_{\Gamma_{i j}^{T}} \zeta_{\mathcal{T}} i j d s\right)^{2} .
\end{aligned}
$$

Thus it holds that

$$
\delta_{3} \leq \eta_{3}\left|z_{\mathcal{T}}\right|_{1,2, \Omega} .
$$

Summarizing the estimates (32) - (35), we obtain

$$
\eta_{n c} \leq\left(C_{1} \eta_{0}+\eta_{1}+\eta_{3}\right)\left|z_{\mathcal{T}}\right|_{1,2, \Omega}+C_{2} \eta_{2}\left\|z_{\mathcal{T}}\right\|_{0,2, \Omega},
$$

where the indicators have the following structure:

$$
\eta_{l}=\left\{\sum_{i \in \Lambda} \eta_{l i}^{2}\right\}^{1 / 2}, \quad l \in\{0,1,2,3\},
$$


where

$$
\begin{aligned}
\eta_{0 i}= & \frac{1}{2}\left\{\sum_{j \in \Lambda_{i}}\left(\mu_{i j}-\frac{1}{m_{i j}} \int_{\Gamma_{i j}} A_{\mathcal{T}} d s\right)^{2}\left(u_{\mathcal{T} i}-u_{\mathcal{T} j}\right)^{2} \frac{m_{i j}}{d_{i j}}\right\}^{1 / 2} \\
& \text { in case of Voronoi diagrams and } \eta_{0 i}=0 \text { in case of Donald diagrams, } \\
\eta_{1 i}= & \left\{\sum_{j \in \Lambda_{i}} \sum_{T \in \mathcal{T}: m_{i j}^{T}>0} h_{T}^{2} \int_{\Omega_{i j}^{T} \cap \Omega_{i}}\left[f-\mathbf{b} \cdot \nabla u_{\mathcal{T}}-c u_{\mathcal{T}}\right]^{2} d x\right\}^{1 / 2}, \\
\eta_{2 i}= & \frac{1}{\sqrt{m_{i}}}\left|\int_{\Omega_{i}}\left[f-f_{i}+(\nabla \cdot \mathbf{b}-c) u_{\mathcal{T}}+c_{i} u_{\mathcal{T} i}\right] d x-\sum_{j \in \Lambda_{i}} u_{\mathcal{T} i} \gamma_{i j} m_{i j}\right|, \\
\eta_{3 i}= & \left\{\frac{d}{2} \sum_{j \in \Lambda_{i}} \sum_{T \in \mathcal{T}: m_{i j}^{T}>0} \frac{d_{i j}}{m_{i j}^{T}}\left(\int_{\Gamma_{i j}^{T}}\left[\left(r_{i j} u_{\mathcal{T} i}+\left(1-r_{i j}\right) u_{\mathcal{T} j}\right) \gamma_{i j}-\left(\nu_{i j} \cdot \mathbf{b}\right) u_{\mathcal{T}}\right] d s\right)^{2}\right\}^{1 / 2} .
\end{aligned}
$$

REMARK 4 (i) We mention that all the indicators $\eta_{l}$ can be rewritten in such a way that the resulting local indicators are related to the elements $T \in \mathcal{T}$.

(ii) It can be shown that the indicators $\eta_{l}$ are order-consistent with the a priori error estimate (Theorem Q in the following sense:

If $f \in W_{q}^{1}(\Omega)$ with some $q>d$ and $u \in W_{2}^{2}(\Omega)$, then there is a constant $C_{c}>0$ such that

$$
\sum_{l=0}^{3} \eta_{l} \leq C_{c} h\left[\|u\|_{2,2}+\|f\|_{1, r}\right],
$$

see [Ang92, Thm. 4] for a special case.

\section{Conclusions and perspectives}

We derived an estimator for measuring simultaneously two types of errors, modeling and discretization errors, with respect to user-defined output functionals. The approach is formulated for stationary nonlinear partial differential equations involving complex models. The main focus was on the consideration of discretization methods which do not possess the property of Galerkin orthogonality. For the example of node-centered finite volume methods, by localization of the estimators we presented local error indicators which allow for local mesh refinement and local model modification.

In future work, the described framework will be the basis for a more detailed analysis of problems with tensor-valued diffusion coefficients and dominating convection and for algorithms which balance the indicators corresponding to the different sources of error.

\section{References}

[AKT98] L. Angermann, P. Knabner, and K. Thiele. An error estimator for a finite volume discretization of density driven flow in porous media. Appl. Numer. Math., 26(12):179-191, 1998 . 
[Ang91] L. Angermann. A modified error estimator of Babuška-Rheinboldt's type for singularly perturbed elliptic problems. In H.-G. Roos, A. Felgenhauer, and L. Angermann, editors, Numerical methods in singularly perturbed problems, pages 1-12. TU Dresden, 1991.

[Ang92] L. Angermann. An a-posteriori estimation for the solution of elliptic boundary value problems by means of upwind FEM. IMA J. Numer. Anal., 12:201-215, 1992.

[Ang95a] L. Angermann. Balanced a-posteriori error estimates for finite volume type discretizations of convection-dominated elliptic problems. Computing, 55(4):305-323, 1995.

[Ang95b] L. Angermann. Error estimates for the finite-element solution of an elliptic singularly perturbed problem. IMA J. Numer. Anal., 15:161-196, 1995.

[Ang00] L. Angermann. A finite element method for the numerical solution of convectiondominated anisotropic diffusion equations. Numer. Math., 85(2):175-195, 2000.

[Ang06] L. Angermann. Transport-stabilized semidiscretizations of the incompressible Navier-Stokes equations. Comput. Methods Appl. Math., 6(3):239-263, 2006.

[Ang10] L. Angermann. Residual type a posteriori error estimates for upwinding finite volume approximations of elliptic boundary value problems. Mathematik-Bericht 2010/1, Institut für Mathematik, Technische Universität Clausthal, 2010.

[AO00] M. Ainsworth and J.T. Oden. A posteriori error estimation in finite element analysis. Wiley, New York, 2000.

[AS06] S.N. Antontsev and S.I. Shmarev. Existence and uniqueness of solutions of degenerate parabolic equations with variable exponents of nonlinearity. Fundam. Prikl. Mat., 12(4):3-19, 2006. Translation in J. of Mathematical Sciences, 150(5):22892301, 2008.

[AW05] L. Angermann and S. Wang. Multidimensional exponentially fitted simplicial finite elements for convection-diffusion equations with tensor-valued diffusion. Calcolo, 42(2):71-91, 2005.

[B0̈8] K. Böhmer. On finite element methods for fully nonlinear elliptic equations of second order. SIAM J. Numer. Anal., 46(3):1212-1249, 2008.

[BE03] M. Braack and A. Ern. A posteriori control of modeling errors and discretization errors. Multiscale Model. Simul., 1(2):221-238 (electronic), 2003.

[BK10] L. Beilina and M.V. Klibanov. A posteriori error estimates for the adaptivity technique for the Tikhonov functional and global convergence for a coefficient inverse problem. Inverse Problems, 26(4):045012, 27, 2010.

[BR78] I. Babuška and W.C. Rheinboldt. Error estimates for adaptive finite element computation. SIAM J. Numer. Anal., 15(4):736-754, 1978. 
[BR01] R. Becker and R. Rannacher. An optimal control approach to a posteriori error estimation in finite element methods. In Acta Numerica, pages 1-102. Cambridge University Press, Cambridge, 2001.

[BR03] W. Bangerth and R. Rannacher. Adaptive finite element methods for differential equations. Lectures in Mathematics ETH Zürich. Birkhäuser Verlag, Basel, 2003.

[BT81] K. Baba and M. Tabata. On a conservative upwind finite element scheme for convective diffusion equations. RAIRO Anal. Numér., 15(1):3-25, 1981.

[Cia78] P.G. Ciarlet. The finite element method for elliptic problems. North-Holland, Amsterdam-New York-Oxford, 1978. Reprinted in 2002 by SIAM, Philadelphia.

[CL05] P. Chatzipantelidis and R. D. Lazarov. Error estimates for a finite volume element method for elliptic PDEs in nonconvex polygonal domains. SIAM J. Numer. Anal., 42(5):1932-1958 (electronic), 2005.

[EFG06] R. Eymard, J. Fuhrmann, and K. Gärtner. A finite volume scheme for nonlinear parabolic equations derived from one-dimensional local Dirichlet problems. Numer. Math., 102(3):463-495, 2006.

[FL01] J. Fuhrmann and H. Langmach. Stability and existence of solutions of timeimplicit finite volume schemes for viscous nonlinear conservation laws. Appl. Numer. Math., 37(1-2):201-230, 2001.

[Ike83] T. Ikeda. Maximum principle in finite element models for convection-diffusion phenomena. North-Holland, Amsterdam-New York-Oxford, 1983.

[KA03] P. Knabner and L. Angermann. Numerical methods for elliptic and parabolic partial differential equations. Texts in Applied Mathematics, Vol. 44. SpringerVerlag, New York, 2003.

$\left[\mathrm{MCL}^{+} 83\right]$ B.J. McCartin, J.R. Caspar, R.E. La Barre, G.A. Peterson, and R.H. Hobbs. Steady state numerical analysis of single carrier two dimensional semiconductor devices using the control area approximation. In Proceedings of the NASECODE III conference, pages 185-190, Dublin, 1983. Boole Press.

[OV00] J.T. Oden and K.S. Vemaganti. Estimation of local modeling error and goaloriented adaptive modeling of heterogeneous materials. I. Error estimates and adaptive algorithms. J. Comput. Phys., 164(1):22-47, 2000.

[Ran05] R. Rannacher. Adaptive finite element methods in flow computations. In Z.-C. Shi, Z. Chen, T. Tang, and D. Yu, editors, Recent Advances in Adaptive Computation. Contemporary Mathematics, vol. 383, pages 183-176. AMS, Providence, Rhode Island, 2005.

[Ris86] U. Risch. Ein hybrides upwind-FEM-Verfahren und dessen Anwendung auf schwach gekoppelte elliptische Differentialgleichungssysteme mit dominanter Konvektion. Dissertation, TH Magdeburg, 1986.

[Ris90] U. Risch. An upwind finite element method for singularly perturbed elliptic problems and local estimates in the $L_{\infty}$-norm. $M^{2} A N, 24(2): 235-264,1990$. 
[Sam65] A.A. Samarskij. Monotonic difference schemes for elliptic and parabolic equations in the case of a non-selfadjoint elliptic operator. U.S.S.R. Comput. Maths. Math. Physics, 5(3):212-217, 1965.

[Thi99] K. Thiele. Adaptive finite volume discretization of density driven flows in porous media. Dissertation, Naturwissenschaftliche Fakultät I, Universität ErlangenNürnberg, 1999.

[VO01] K.S. Vemaganti and J.T. Oden. Estimation of local modeling error and goaloriented adaptive modeling of heterogeneous materials. II. A computational environment for adaptive modeling of heterogeneous elastic solids. Comput. Methods Appl. Mech. Engrg., 190(46-47):6089-6124, 2001.

[Voh08] M. Vohralík. Residual flux-based a posteriori error estimates for finite volume and related locally conservative methods. Numer. Math., 111(1):121-158, 2008. 